\title{
MHD simulations of ram pressure stripping of a disk galaxy
}

\author{
Mariana Ramos-Martínez ${ }^{1 \star}$, Gilberto C. Gómez ${ }^{1}$ and Ángeles Pérez-Villegas ${ }^{2,3}$ \\ ${ }^{1}$ Instituto de Radioastronomía y Astrofísica, Universidad Nacional Autónoma de México, Apdo. Postal 3-72, Morelia Mich. 58089, México \\ ${ }^{2}$ Max-Planck-Institut für Extraterrestrische Physik, Gießenbachstraße, D-85741, Garching, Germany \\ ${ }^{3}$ Universidade de São Paulo, IAG, Rua do Matão 1226, Cidade Universitária, São Paulo 05508-900, Brazil
}

Accepted XXX. Received YYY; in original form ZZZ

\begin{abstract}
The removal of the interstellar medium (ISM) of disk galaxies through ram pressure stripping (RPS) has been extensively studied in numerous simulations. Nevertheless, the role of magnetic fields (MF) on the gas dynamics in this process has been hardly studied, although the MF influence on the large-scale disk structure is well established. With this in mind, we present a 3D magnetohydrodynamic (MHD) simulation of face-on RPS of a disk galaxy to study the impact of the galactic MF in the gas stripping. The main effect of including a galactic MF is a flared disk. When the intracluster medium (ICM) wind hits this flared disk, oblique shocks are produced at the interaction interface, where the ISM is compressed, generating a gas inflow from large radii towards the central regions of the galaxy. This inflow is observed for $\sim 150 \mathrm{Myr}$ and may supply the central parts of the galaxy with material for star formation while the outskirts of the disk are being stripped of gas, thus the oblique shocks can induce and enhance the star formation in the remaining disk. We also observed that the MF alters the shape and structure of the swept gas, giving a smooth appearance in the magnetized case and clumpier and filamentary-like morphology in the hydro case. Finally, we estimated the truncation radius expected for our models using the Gunn-Gott criterion and found that is in agreement with the simulations.
\end{abstract}

Key words: MHD - galaxies: ISM - galaxies: individual: M33 - galaxies: magnetic fields - galaxies: evolution.

\section{INTRODUCTION}

Lenticular galaxies (S0s) are objects that lie between the elliptical and spiral galaxies in the Hubble sequence. The S0s share properties with both types of galaxies, that is, an old stellar population like ellipticals and stellar disks like spirals. Lenticulars also have prominent bulges (Simien \& de Vaucouleurs 1986), low gas content (Gallagher, Faber \& Balick 1975) and some observations show that the last star formation episode took place at the bulge (Prochaska Chamberlain et al. 2011; Sil'chenko 2006; Sil'chenko et al. 2012; Bedregal 2012; Johnston et al. 2012, 2014, but see Katkov et al. 2015).

The well studied environmental density-galactic morphology relationship in clusters of galaxies (Dressler 1980) states that late-type galaxies (spirals) are more frequently found in the outskirts of clusters, while early-type galaxies (ellipticals and S0s) are more abundant in the central regions. In the case of groups of galaxies, a similar

* E-mail: m.ramos@irya.unam.mx (MRM) trend has been observed (Postman \& Geller 1984). Additionally, in cluster galaxies, the fraction of spirals increases with increasing redshift $z$, whilst the S0s fraction decreases (Dressler et al. 1997; Fasano et al. 2000). On the other hand, when properties of spiral galaxies in clusters and those in the field are compared (Boselli \& Gavazzi 2006 and references therein), cluster spirals are HI deficient and such deficiency increases towards the cluster centre. Also, cluster galaxies show a lower star formation rate (SFR) associated with the lack of HI, and they are redder than field galaxies, which indicates the former form stars passively (Butcher \& Oemler 1978). Late-type galaxies also follow more radially elongated orbits than early-type, suggesting they are free-falling into the cluster (Giraud 1986; Dressler 1986; Vollmer et al. 2001; Biviano \& Katgert 2004; Aguerri et al. 2017). Lastly, cluster galaxies show an increase in radio-continuum emission, probably due to an enhancement in the magnetic field (MF) intensity, possibly caused by compression (Scodeggio \& Gavazzi 1993; Rengarajan et al. 1997).

These observations point to one or more mechanisms 
that act in the environment of clusters and groups, stripping the galactic interstellar medium (ISM) from the disks or increasing its consumption rate so that the star formation shuts down and a change in disk colour is produced. Therefore, the idea that spirals are the progenitors of lenticular galaxies has been proposed, suggesting that the study of S0s may help us understand the impact of environment on the evolution of disk galaxies.

In clusters, the main mechanisms proposed to explain the transformation of a spiral galaxy into an S0 are:

- Ram pressure stripping (RPS, Gunn \& Gott 1972): when a galaxy falls into the cluster centre, the hot intracluster medium (ICM) exerts an hydrodynamic pressure on the ISM of the galaxy and, if this pressure exceeds the gravitational force of the disk (Gunn-Gott criterion), then the ISM is stripped off the galaxy.

- Galaxy harassment (Moore et al. 1996): close and frequent encounters between galaxies occurring at high velocities, at a rate of one encounter per $1 \mathrm{Gyr}$, may increase the SFR, rapidly exhausting the gas supply and eventually leading to a redder disk. These interactions will alter the galactic morphology by dynamically heating the disk.

- Starvation (Larson et al. 1980): the galaxy loses the envelope of hot gas that supplies the disk's gas reservoir, so the ISM is consumed and the star formation shuts down.

There are also other mechanisms that may act in groups of galaxies that can modify the galactic morphology, such as tidal interactions (Icke 1985), and major (Toomre \& Toomre 1972; Borlaff et al. 2014) and minor mergers (Aguerri et al. 2001; Tapia et al. 2014). Nevertheless, these processes are not exclusive, that is, more than one might operate at the same time. Comparing these mechanisms, Boselli \& Gavazzi (2006) conclude that RPS is the most appropriate to explain the differences observed in between spirals of clusters and those in the field, since RPS removes the gas from the galaxies producing a change in the SFR and colour. Also, RPS is efficient and inevitable near the cluster centre and may alter indirectly the morphology of the disks (if a galaxy loses its gas, the stellar disk is dynamically heated, leading to a thicker disk, Farouki \& Shapiro 1980; Sellwood \& Carlberg 1984; Fuchs \& von Linden 1998; Bekki et al. 2002; Elmegreen et al. 2002).

Multiwavelength observations have shown several cluster galaxies that are good candidates to be experiencing RPS (Koopmann \& Kenney 2004; Chung et al. 2009; Yagi et al. 2010; Kenney et al. 2014; Boselli et al. 2014; Bekki 2014), since they show truncated gaseous disks and in some cases gas tails, while the stellar disk remains unperturbed. Cayatte et al. (1990) performed a survey of HI for spiral galaxies in the Virgo Cluster where they found that small HI disks lie almost exclusively in the cluster centre in galaxies with high velocities with respect to the cluster mean velocity, which make it possible they lost their gas through ram pressure stripping. Moreover, they observed that galaxies affected by RPS have shown nuclear activity. This could be since the gas pushed to the centre of the galaxy and the compression exerted by the ICM enhances the star formation. Also, Poggianti et al. (2016) presented an atlas of galaxies at low redshift that are being stripped of their ISM, with candidates found at all cluster centric distances that showed an enhanced SFR compared to non-candidates of the same mass. This points to the idea that RPS can induce and enhance the star formation.

A good example of a galaxy subject to RPS is NGC 4522 in the Virgo cluster. This is the most studied case of a galaxy losing its ISM by this mechanism (Vollmer et al. 2000, 2004, 2006, 2008; Abramson \& Kenney 2014; Abramson et al. 2016; Stein et al. 2017) and is possibly in the process of transforming into an S0, since it shows a truncated disk in $\mathrm{HI}$ with a $3 \mathrm{kpc}$ radius and a $\sim 3$ kpc-length gas tail observed in HI (Kenney et al. 2004) and $\mathrm{H} \alpha$ (Kenney \& Koopmann 1999). Also, in the Abell 3627 cluster, the galaxy ESO 137-001 is stripped by the hot ICM (Sun et al. 2006, 2007; Sivanandam et al. 2010; Fumagalli et al. 2014; Jáchym et al. 2014; Fossati et al. 2016). ESO 137-001 presents an $80 \mathrm{kpc}$ long, double X-ray gas tail (Sun et al. 2006), with some HII regions embedded within the tail (Sun et al. 2010), indicating that star formation can go on within the ISM stripped out of the galaxy. Later, in the same cluster, another X-ray gas tail was detected (ESO 137-002), with a double $\mathrm{H} \alpha$ tail (Zhang et al. 2013).

The ICM-ISM interaction through the RPS has been studied extensively for years. A wide variety of models have been developed with different methods and techniques. The first models were performed under the assumption of a constant ICM wind, using smoothed particle hydrodynamics (SPH; Abadi et al. 1999; Schulz \& Struck 2001) and grid codes (Quilis et al. 2000; Roediger \& Hensler 2005; Roediger \& Brüggen 2006; Roediger, Brüggen \& Hoeft 2006). These models were in good agreement with the Gunn-Gott estimation for the disk truncation radius. Other simulations were done varying the inclination angle of the disk with respect to the wind direction (Vollmer et al. 2006; Roediger \& Brüggen 2006; Jáchym et al. 2009). Yet other models added a variable ICM wind, so the RPS mechanism is not constant (Roediger \& Brüggen 2007, 2008; Vollmer et al. 2001, with a sticky-particle code). Another extension to the RPS models included a multiphase gas disk (Quilis et al. 2000; Tonnesen \& Bryan 2009, 2010), where the low-density gas is stripped more easily from the galaxy, but the mass loss of the ISM is not so different from homogeneous disk models. Some other works included star formation, which showed an increase in the star formation in central regions of the target galaxies (Schulz \& Struck 2001; Vollmer et al. 2001) and sometimes stars were formed in the gas tails (Bekki \& Couch 2003; Kronberger et al. 2008; Kapferer et al. 2008; Steinhauser et al. 2012; Tonnesen \& Bryan 2012).

Despite the huge variety of RPS models, there are very few including MF. MF have been observed in galaxies from polarized emission, mainly in radio frequencies, and Faraday rotation. MF in spirals have an ordered component, i.e. with a constant and coherent direction, and a random or turbulent component that has been amplified and tangled by turbulent gas flows (Beck 2005, Beck \& Wielebinski 2013 and references therein). Combining information obtained with different techniques, it is possible to develop a model for the 3D structure of MF in galactic disks. In spirals, the average total field strength is $\sim 9 \mu \mathrm{G}$ (Niklas 1995) and the regular field strength is $1-5 \mu \mathrm{G}$ (Beck \& Wielebinski 2013), in radio-faint galaxies like M31 and M33 the total field is $6 \mu \mathrm{G}$ (Gießübel 2012; Tabatabaei et al. 2008), 
in gas rich spiral galaxies the total field is $20-30 \mu \mathrm{G}$ (Fletcher et al. 2011; Frick et al. 2016), for bright galaxies $\sim 17 \mu \mathrm{G}$ (Fletcher 2010), in blue compact dwarf galaxies $10-20 \mu \mathrm{G}$ (Klein et al. 1991) and the strongest total fields are found in starburst and barred galaxies with $50-100 \mu \mathrm{G}$ (Adebahr et al. 2013; Chyży \& Beck 2004; Beck et al. 2005). Since the degree of polarization on average is low in the spiral arms, the random field is assumed to be stronger, up to five times the intensity of the ordered field, whilst in the interarm region the degree of polarization is higher, hence the ordered field should dominate. Additionally, it has been observed that the ordered MF shows a spiral pattern that is offset from the spiral arms of gas and stars (Beck 2005).

Ruszkowski et al. (2014) presented simulations of RPS with a magnetized ICM and found that the MF can affect the morphology of the stripped gas tail, since they observed narrower tails than in purely hydrodynamic (HD) simulations. Pfrommer \& Dursi (2010) also showed magnetohydrodynamics (MHD) simulations in which the galaxies are moving in a magnetized ICM. The galaxies in their simulations swept the field lines where polarized radiation is generated. This is used to map the orientation of the MF in clusters, e.g. Virgo cluster. In these cases, the MF has been implemented only in the ICM and not in the disks. Some examples of models with magnetized disks are Vollmer et al. (2006, 2007); Soida et al. (2006), where they used the method of Otmianowska-Mazur \& Vollmer (2003) where the MF is evolved via the induction equation using a grid code with the velocity field of the particles, so that the MF is advected with the gas. These simulations of RPS have been carried out first with a sticky particle code, and then a toroidal configuration of the MF is given to the galaxy. Even if the effect of the MF over the gas dynamics has not been taken into account, this method has been useful to explain the polarized radiation in radio that is observed in some galaxies that may be affected by the RPS, as in the case of NGC 4522 (Vollmer et al. 2006).

Additionally, Tonnesen \& Stone (2014) performed MHD simulations for the RPS including galactic MF, but the ICM was not magnetized. They found that MF do not alter or dramatically change the stripping rate of the gas disk compared to pure HD simulations. Nevertheless, the MF have an impact in the mixing of gas throughout the tail, since inhibits the mixing of the gas tail with the ICM, the unmixed gas survives at large distances from the disk. Besides, the RPS may help magnetize the ICM up to a few $\mu G$.

Here, we present MHD simulations of ram pressure stripping of a disk galaxy under the wind-tunnel approximation, for a face-on geometry. Additionally, we performed two purely HD runs to compare with the magnetized case and analyze the impact that the galactic MF has in the stripping of the disk. In $\S 2$ we present the initial set up for the simulations, in $\S 3$ we describe the resulting gas and MF distribution, and in $\S 4$ we discuss our conclusions.

\section{MODEL}

We set up a magnetized disk in rotational equilibrium in a fixed gravitational potential. We used the MHD code RAMSES (Teyssier 2002), which is an adaptive mesh refinement

\begin{tabular}{cc}
\hline Bulge & $\mathrm{M}_{1}=1.39 \times 10^{9} \mathrm{M}_{\odot}$ \\
& $\mathrm{b}_{1}=0.85 \mathrm{kpc}$ \\
\hline Disk & $\mathrm{M}_{2}=1.62 \times 10^{10} \mathrm{M}_{\odot}$ \\
& $\mathrm{a}_{2}=3.0 \mathrm{kpc}$ \\
& $\mathrm{b}_{2}=1.0 \mathrm{kpc}$ \\
\hline Halo & $\mathrm{M}_{3}=6.96 \times 10^{10} \mathrm{M}_{\odot}$ \\
& $\mathrm{a}_{3}=16.0 \mathrm{kpc}$ \\
\hline
\end{tabular}

Table 1. Length scale and mass parameters of the gravitational potential, as adjusted to approximate M33's rotation curve. $\mathrm{M}_{1}$ and $\mathrm{M}_{2}$ represent the total mass of the bulge and disk, respectively, while $\mathrm{M}_{3}$ is a mass factor for the halo, where its total mass is obtained up to a cutoff radius.

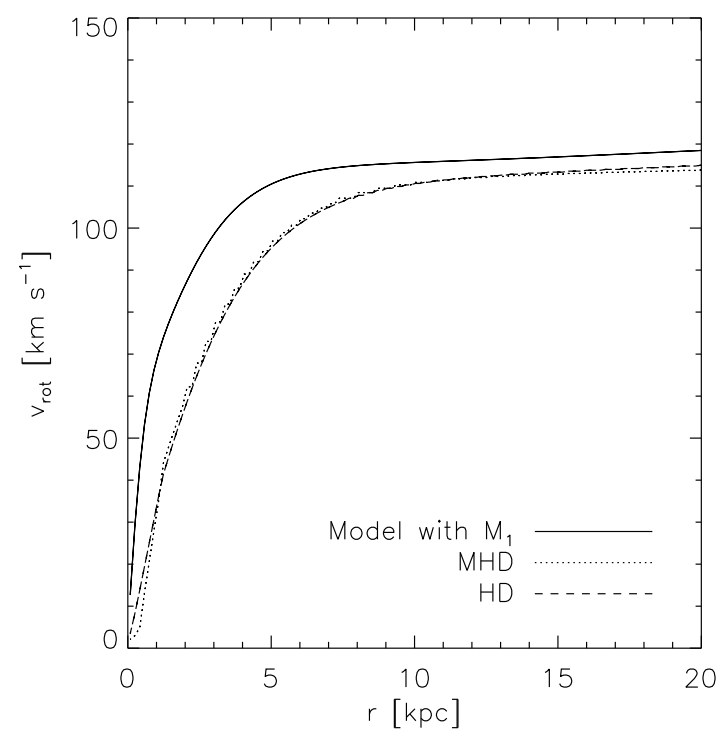

Figure 1. Rotation curve for the M33-like galaxy obtained from the gravitational potential with the values from Table 1 (solid line) and velocity profile for the MHD (dotted line) and the HD (dashed line) simulations without the bulge contribution $\left(M_{1}=\right.$ $0)$.

code, so we can have more refinement of cells in the desired regions, and allows us to add $\mathrm{MF}$ in the simulations. The models were performed in 3D with 11 refinement levels, for a resolution equivalent to $(2048)^{3}$ cells, in a box of $120 \mathrm{kpc}$ in each direction.

\subsection{Initial Conditions}

The gravitational potential used for our galaxy is based on the model of Allen \& Santillán (1991), which is an analytic and simple potential that can reproduce the rotation curve of the Milky Way and is composed by a spherical central bulge, a Miyamoto-Nagai disk and a massive spherical halo. This potential model can be easily modified to approximate the rotation curves of other galaxies. For this work, we modeled an M33-like galaxy, which is a late type and low-luminosity spiral galaxy. We modified the mass and scale parameters to the values shown in Table 1 to model the rotation curve 
of the M33 galaxy (see figure 1, solid line) as reported by Corbelli (2003). Nevertheless, for the simulations presented in this work, we removed the galactic bulge component of the potential $\left(M_{1}=0\right)$ since it generated a large potential gradient in the $z$-direction (perpendicular to the galactic disk) for small radii that generated problems for our initial setup procedure (described below). Regardless, this should have little impact on our conclusions, specially since the M33 bulge's mass is small. The velocity profile used for the simulations, both with and without magnetic fields, is also presented in figure 1.

For the initial conditions, we use a method similar to Gómez \& Cox (2002). First, we define the radial density and velocity profile in the galactic mid-plane, assuming that the gas disk is in rotational equilibrium with the gravitational force, the total pressure gradient, and the magnetic tension,

$\frac{v_{\phi}^{2}(r, z)}{r}=\frac{\partial \Phi}{\partial r}+\frac{1}{\rho(r, z)}\left[\frac{\partial P}{\partial r}+\frac{2 P_{B}(r, z)}{r}\right]$,

where the total pressure $P$ is the sum of the thermal $\left(P_{t h}=\right.$ $c_{s}^{2} \rho(r, z)$, with $c_{s}$ the sound speed) and the magnetic $\left(P_{B}\right)$ pressures. The magnetic pressure has two components, $P_{B}=$ $P_{B, \text { inner }}+P_{B, \text { outer }}$, with

$$
\begin{aligned}
P_{B, \text { inner }} & =P_{B 0}\left[1-\operatorname{erf}\left(\frac{R}{r_{b}}\right)\right] \text { and } \\
P_{B, \text { outer }} & =\frac{P_{B 0} n}{\left(n+n_{c}\right)},
\end{aligned}
$$

where $R=\sqrt{r^{2}+z^{2}}, r_{b}=b_{1} / 3$ (see table 1 ), $P_{B 0}=$ $1.75 \times 10^{-13} \mathrm{dyn} \mathrm{cm}^{-2}$ and $n_{c}=0.04 \mathrm{~cm}^{-3}$. With these expressions for the total pressure and eq. (1), a given midplane density (or velocity) profile uniquely defines the velocity (density) profile. In the bulge, the rotation curve resembles a rigid body, and so we define the rotation velocity linearly increasing with radius,

$v_{\phi}(r, 0)=v_{\phi}\left(b_{1}, 0\right)\left[\frac{r}{b_{1}}\right]$,

where $v_{\phi}\left(b_{1}, 0\right)$ is the circular velocity obtained from the gravitational potential in $r=b_{1}$ and $z=0$. Then, the density profile in the midplane is given by,

$\frac{\partial \rho}{\partial r}=\frac{\rho\left(v_{\phi}^{2}-\partial \Phi / \partial r\right)+\left(P_{B 0} / r_{b}\right) e^{-\left(R / r_{b}\right)^{2}}}{\left[c_{s}^{2}+P_{B 0} \rho_{c} /\left(\rho+\rho_{c}\right)^{2}\right]}$,

which is integrated from $r=0$ to $b_{1}$.

For $r>b_{1}$ we do the converse: we define the density profile as exponentially decreasing at the mid-plane,

$\rho(r, 0)=\rho_{0} \exp \left[-\left(r-b_{1}\right) / h_{r}\right]$,

where $h_{r}=6 \mathrm{kpc}$ and $\rho_{0}$ is the value found at $r=b_{1}$ from eq. (5).

Once the mid-plane density is calculated, the distribution at $z \neq 0$ is found by assuming magnetohydrostatic equilibrium and an isothermal equation of state,

$\frac{\partial P}{\partial z}=-\rho \frac{\partial \Phi}{\partial z}$ where again $P=P_{t h}+P_{B}$. By substituting the magnetic pressure components (eqs. 2 and 3 ) and the equation of state it follows

$\frac{\partial \rho}{\partial z}=\frac{-\rho \partial \Phi / \partial z+\left(P_{B 0} / r_{b}\right) \exp \left[-\left(R / r_{b}\right)^{2}\right]}{\left[c_{s}^{2}+P_{B 0} \rho_{c} /\left(\rho+\rho_{c}\right)^{2}\right]}$,

which is integrated along the $z$ coordinate to obtain the vertical density profile at any radius $r$.

The rotation velocity above the midplane is given by (Gómez \& Cox 2002):

$v_{\phi}^{2}(r, z)=v_{\phi}^{2}(r, 0)-v_{A}^{2}(r, 0)+v_{A}^{2}(r, z)$,

where $v_{A}$ is the Alfvén velocity $\left(v_{A}=\sqrt{2 P_{B} / \rho}\right)$.

Figure 2 shows a density map for the initial condition of the disk at $y=0$, both for a magnetized and a purely HD $\left(P_{B 0}=0\right)$ cases. It can be seen that, for the magnetized case, the disk is thicker in the outskirts than in the central region, that is, the galactic disk flares in the presence of the MF. Additionally, the scale height of the MHD disk is larger than in HD one. When solving the equation of hydrostatic equilibrium, the MF changes the compressibility of the gas, thus increasing the surface density $\Sigma$ for the gravitational potential and midplane density used, which results in a heavier disk compared to the HD model. For this reason, we performed another HD simulation with surface density similar to that in the magnetized disk model. We will refer to this as the heavy disk model. To obtain the density distribution of the heavy disk, we solve again the equations (5) and (7), increasing the initial value of $\rho$ one order of magnitude over original HD model, thus increasing $\rho_{H D}(z=0)$ results in $\Sigma_{\text {heavy }} \sim 1.5 \Sigma_{M H D}$. The initial density distribution for the heavy model is also presented in figure 2 .

\subsection{Galactic magnetic field}

In the setup described above for the MHD model, the MF has two components (eqs. 2 and 3 ). While the outer component $\left(r>b_{1}\right)$ is purely toroidal, the inner one is random. For the random inner component $\left(r<b_{1}\right)$, we defined the vector potential A with,

$$
\begin{aligned}
& A_{x}=A_{0} \cos \phi_{r} \sin \theta_{r} f(z) \\
& A_{y}=A_{0} \sin \phi_{r} \sin \theta_{r} f(z) \\
& A_{z}=A_{0} \cos \theta_{r} f(z)
\end{aligned}
$$

where the angles $\phi_{r}$ and $\theta_{r}$ where obtained randomly and $A_{0}$ is drawn from a normal distribution with dispersion equal to $\sqrt{8 \pi P_{B 0}}$.

The function $f(z)=\operatorname{sech}^{2}\left(z / z_{h}\right)$, with $z_{h}=150 \mathrm{pc}$, modulates the vector potential so its magnitude has the same scale height as the density in the bulge. Once the components for the vector potential are calculated, it is smoothed in order to avoid large fluctuations. Finally, the $\mathrm{MF}$ is calculated $\mathbf{B}_{\text {inner }}=\nabla \times \mathbf{A}$. For the rest of the disk $\left(r>b_{1}\right)$, the MF in the setup follows a toroidal configuration, with its strength given by the gas density (eq. 3). Figure 3 shows the initial intensity of the MF with arrows overlaid representing the field lines for the MHD model. 


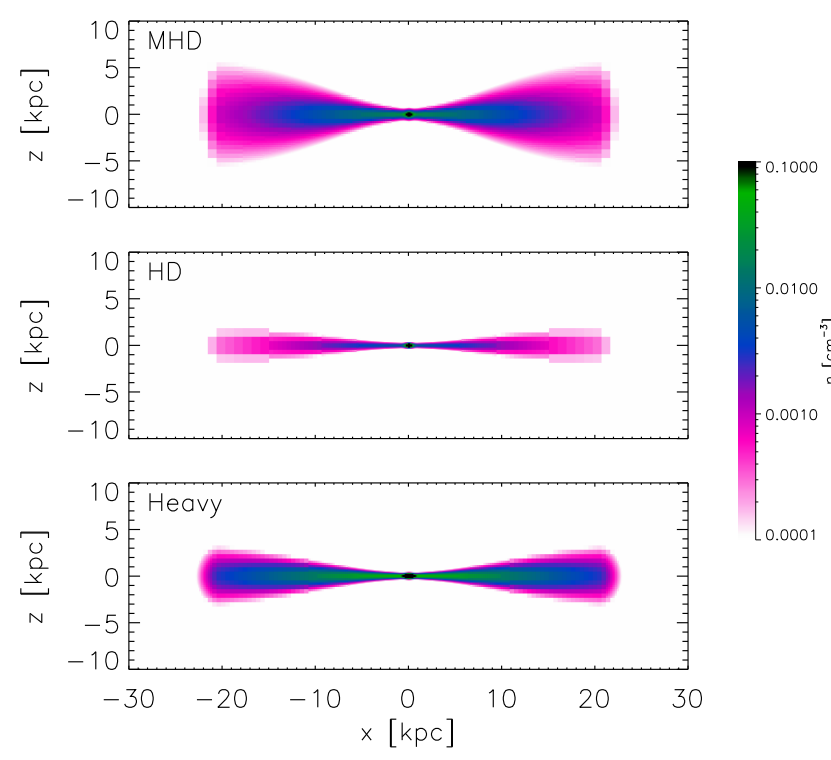

Figure 2. Density slices of the initial condition for the MHD (top), the HD (center) and the heavy (bottom) models. Colours show the gas number density at the $y=0$ plane.

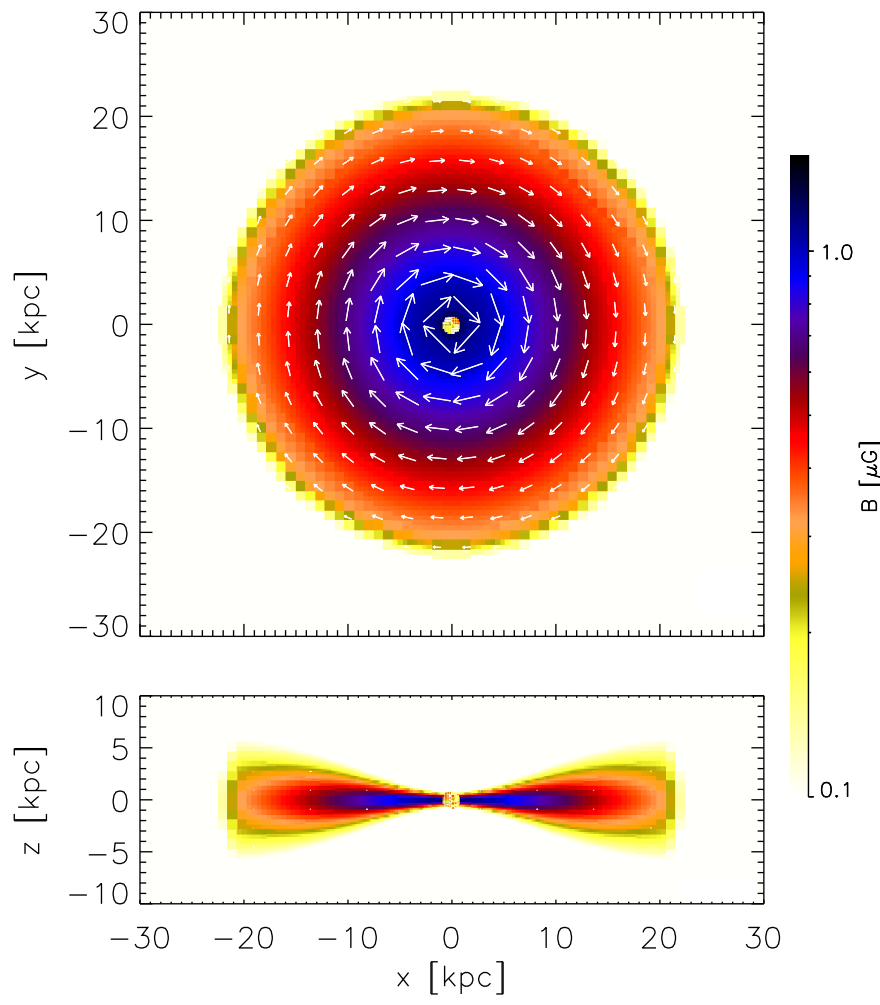

Figure 3. Slices of the MF intensity for the MHD model at the initial condition in a $z=0$ (top) and $y=0$ (bottom) cut. The MF strength is colour-coded in log-scale.

\subsection{ICM wind}

To simulate the ICM-ISM interaction, we worked under the wind-tunnel approximation, this is, we place the galaxy at rest and the ICM flows towards the disk face-on. The ICM wind is unmagnetized and has the same parameters for all models: the wind starts at $z=-10 \mathrm{kpc}$ and moves in the $+z$ direction with density $n_{\mathrm{ICM}}=10^{-5} \mathrm{~cm}^{-3}$ and a velocity that increases linearly in time, from $300 \mathrm{~km} \mathrm{~s}^{-1}$ to $760 \mathrm{~km} \mathrm{~s}^{-1}$ at the end of the simulation, at $500 \mathrm{Myr}$. All the computational boundaries are outflowing, except at the bottom where the wind flows inward.

\section{RESULTS}

\subsection{Model evolution}

Figure 4 shows the evolution of the models: the magnetized and both HD and heavy hydro models, in maps of projected density. The first row corresponds to a time $t=90 \mathrm{Myr}$. It can be seen that the wind is starting to interact with the disks. In the MHD run (left column), oblique shocks appear on the side of the disk that is facing the ICM wind (at $z<0$ ), because our disk flares in the presence of the galactic MF, giving it a "bow tie" shape. The oblique shocks lead gas of the external parts of the disk towards the galactic centre and continues for another $\sim 150 \mathrm{Myr}$ more until the ICM wind finally surpasses the gravitational force of the disk and starts to sweep the ISM (see $\S 3.3$ ). Since the HD disk (central column) does not flare as much as the MHD one, the ISM-ICM interaction is different. At $90 \mathrm{Myr}$, in the HD model the wind perturbs the gaseous disk and displaces it from the $z=0$ midplane. The background gravitational force pulls back the gas to its original position, mainly in the inner region of the disk, whilst the outer parts of the disk are still being swept by the wind. In the heavy model, most of the gas disk initially at $z<0$ is compressed and moved by the wind to a height $z \sim 0$, changing the disk symmetry but to a smaller extent than in the HD case.

At $t=190 \mathrm{Myr}$ (second row), for the MHD run, most of the gas that lied below the galactic midplane was swept by the wind which in turn starts to erode the disk at large radii, where the ram pressure exceeds the gravitational force of the galaxy (see §3.2). The inner region of the disk $(r<$ $5 \mathrm{kpc})$ is slightly perturbed, with small variations in the $z$ direction, since the gravitational force tries to keep the disk in the equilibrium position. As a result, the gas moves up and down. These fluctuations in $z$ at small radii occur at earlier times in the HD model but it is basically the same behavior: gas at large $r$ is swept by the wind whilst the gas located near the galactic centre remains bound to the disk. The displaced gas reaches a height of $\sim 5 \mathrm{kpc}$ and $\sim 10 \mathrm{kpc}$ above the galactic midplane, for the MHD and the HD case, respectively. The HD model shows a larger erosion than the MHD, with a gaseous disk of radius $r<5 \mathrm{kpc}$ for the HD run, and $r \sim 20 \mathrm{kpc}$ for the MHD one. The gas of the heavy disk has moved just a few kpc from the midplane, showing almost the same radial extension as the MHD, although the heavy disk is denser near the galactic midplane.

After $310 \mathrm{Myr}$ of evolution, the wind continues flowing and accelerating towards the disk and reaches $v_{\mathrm{ICM}} \sim$ $550 \mathrm{~km} \mathrm{~s}^{-1}$ at $z=0$. For the magnetized disk (third row, 

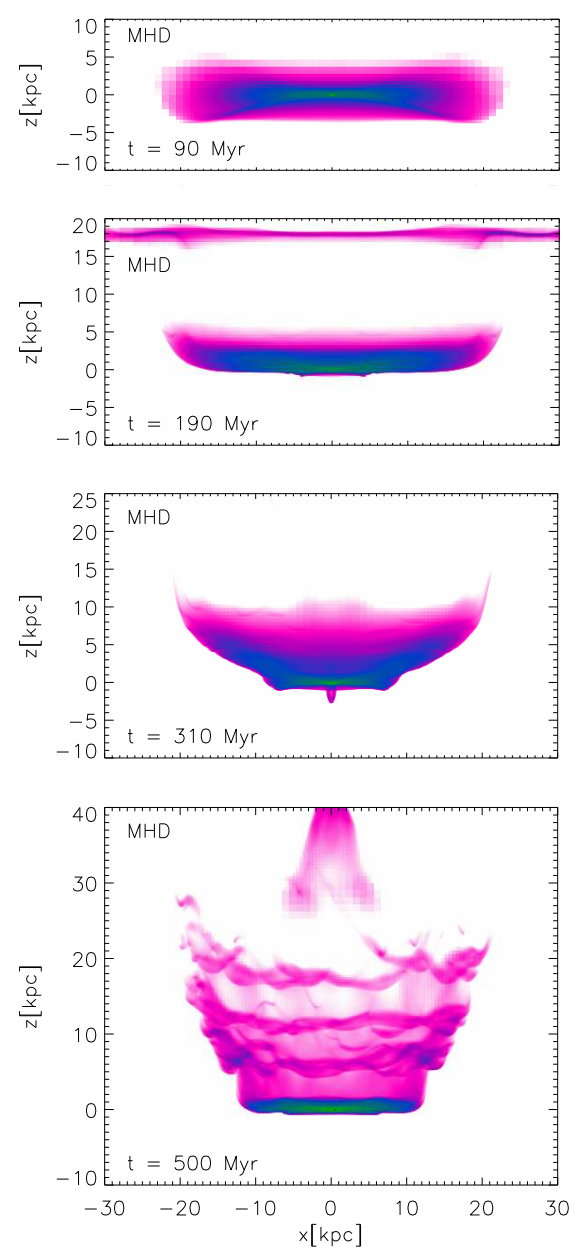
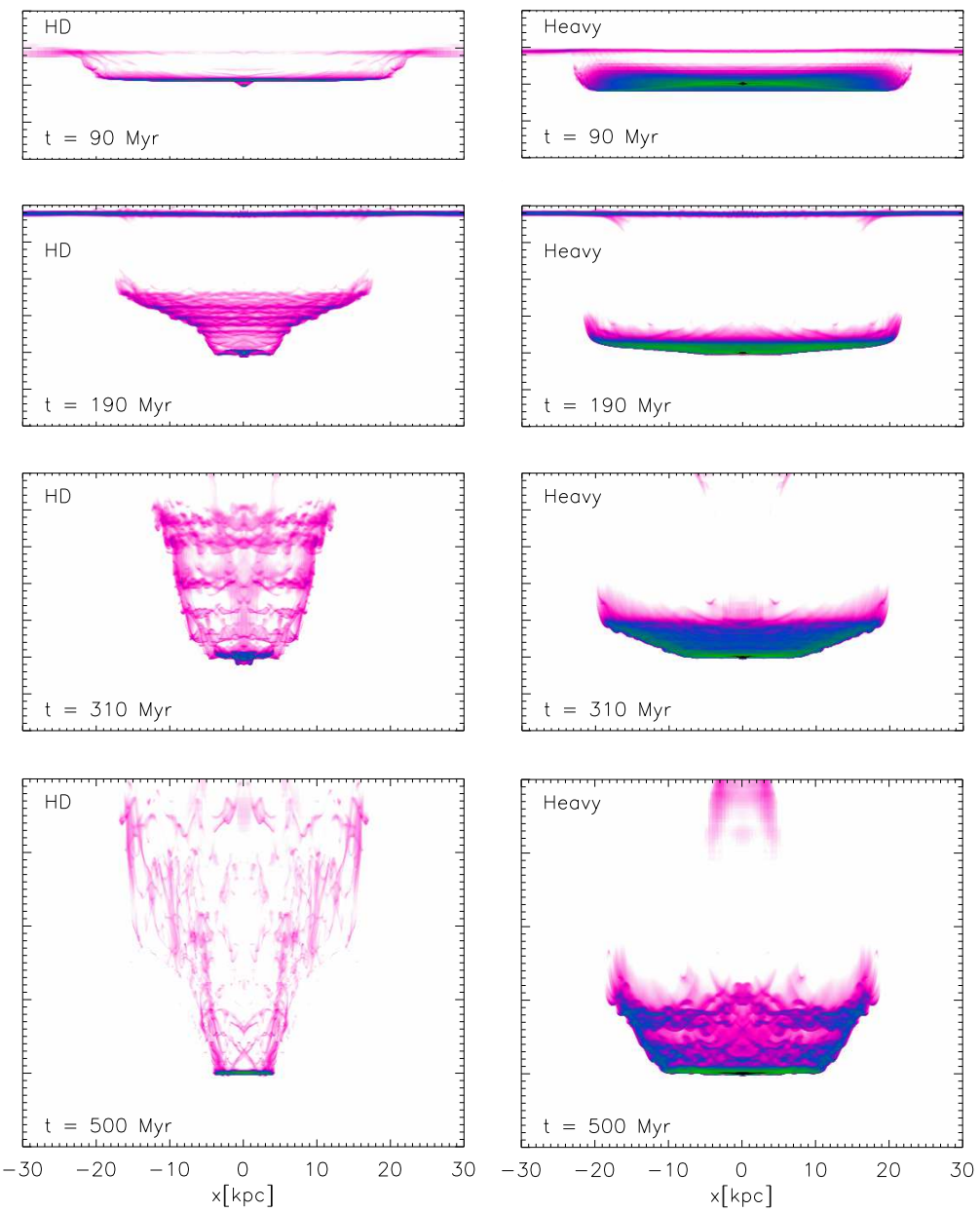

0.0100

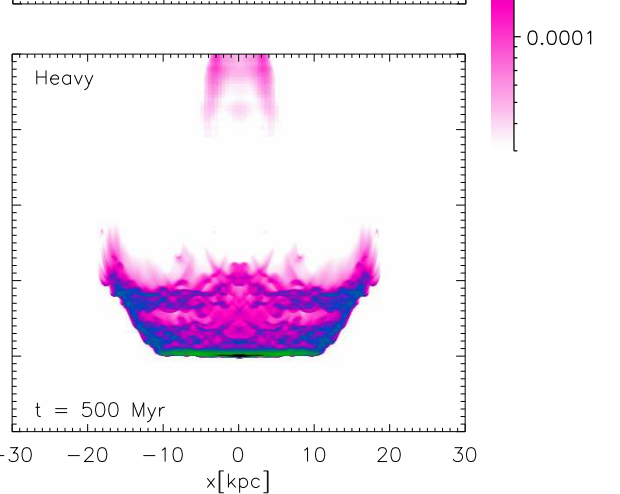

Figure 4. Projected density of the gas along the $y$-axis for the MHD (left column), HD (center) and heavy (right column) runs. Each row corresponds to times $t=90,190,310$ and $500 \mathrm{Myr}$. The ICM wind moves up from bottom of the computational grid. Please note that the $z$-axis limits are different for each time shown.

left), the gas at $r>10 \mathrm{kpc}$ is ripped off of the galaxy, where the ram pressure is stronger than the galactic gravitational force. The swept gas has increased its height, reaching $z \sim 10 \mathrm{kpc}$. There are still some vertical motions in the midplane for $r<10 \mathrm{kpc}$ because the gas in this position is adjusting to the balance between the pressure from the wind and the gravitational force in the disk. This process is also present in the HD run, but the oscillating gas is contained in a smaller radius $(r<7 \mathrm{kpc})$. Additionally, for the HD model, the gas that has been removed from the disk has reached a height of $\sim 20 \mathrm{kpc}$ above the disk midplane. Compared to the magnetized case, the stripped gas has a more diffuse appearance in the HD run, that is, the gas mixes easier with the surrounding, and it is less extended in the radial direction than the MHD case. The HD disk is, at this time, truncated to a radius of $\sim 6 \mathrm{kpc}$. The heavy disk at $t=310$ Myr shows a structure similar to the MHD one: the heavy disk has a radial extension of $r \sim 10 \mathrm{kpc}$ in the midplane, while in the vertical direction the denser gas reaches a height of $z \sim 5 \mathrm{kpc}$, but the less dense gas is farther away the galactic midplane in the MHD model $(z \sim 10 \mathrm{kpc})$.
At $t=500$ Myr the wind has a velocity of $\sim 760 \mathrm{~km} \mathrm{~s}^{-1}$ at $z=0$ for all cases. In the MHD model, the stripped gas reaches a height of $\sim 20 \mathrm{kpc}$ above the galactic midplane. The disk has been truncated to a radius of $\sim 10 \mathrm{kpc}$, which is approximately half of its original size. The dimensions of the displaced gas for the MHD model resembles the one from the HD at $t=310 \mathrm{Myr}$, showing a similar longitude over the midplane, which suggests that the evolution of the MHD simulation is delayed with respect to the HD run, although differences remain in the morphology: in the MHD case, the swept gas has a smooth appearance and is denser at higher $z$ than in the HD case, which indicates that the MF prevents the gas from mixing with the surroundings, similarly as seen in Tonnesen \& Stone (2014). On the other hand, the HD model with $500 \mathrm{Myr}$ of evolution shows a more filamentary and clumpier morphology in the stripped gas, contrary to the smooth appearance that the magnetized gas presents. The HD gas is extended over $\sim 40 \mathrm{kpc}$ above $z=0$, and the remaining disk has radius of $\sim 4 \mathrm{kpc}$, which indicates that this disk has reached a state of equilibrium with the ram pressure, since the gas was rapidly eroded in the first $\sim 200 \mathrm{Myr}$ 
of evolution and the remaining gaseous disk (in $z=0$ ) has the same radial extension until the end of the simulation. The heavy disk has a size similar to the MHD $(r \gtrsim 10 \mathrm{kpc})$ in the midplane $(z=0)$, suggesting that the stripping rate for both disks is approximately the same, whilst the displaced gas for the heavy model has a lower $z$-height. Nevertheless, when the heavy and the HD simulations are compared, the displaced gas of the heavy model resembles the HD case, in that both have a clumpy and filamentary-like structure, with the difference that, in the heavy model, the swept gas is denser because of the initial condition of the gas disk, that is $\rho_{\text {heavy }}>\rho_{H D}\left(\Sigma_{\text {heavy }}>\Sigma_{H D}\right)$ as mentioned in $\S 2.1$, which also results in a slower erosion of the disk.

Comparing the evolution of the three models, the MHD and the heavy model are left with a similar remnant disk, with radius $r \sim 10-12 \mathrm{kpc}$ which is larger than the HD model $(r \sim 4 \mathrm{kpc}$ ) for the same time of evolution. Our results suggest that the stripping rate depends on the MF only through the surface density $\Sigma$ of the disk: a heavier disk (high $\Sigma$ ) is more difficult to erode since the ICM has more material to sweep, even when the gas is farther away from the gravitational potential well, and thus less bound to the galaxy. This is similar to the results presented by Tonnesen \& Stone (2014), where the MHD and the HD disks with the same initial mass do not show a significant difference in the stripping rate. Although the heavy model agrees quite well with the MHD in the rate at which the gaseous disk is removed and the truncation radius (see $\S 3.2$ ), the problem with the heavy disk is that it gives a higher and unrealistic volumetric density $\rho$ in the galactic midplane, because in the absence of the MF, using the same potential to solve the hydrostatic requires a high value of $\rho$ to obtain the same $\Sigma$ of the magnetized case. Nevertheless this heavy model is useful to investigate the dependence of the stripping with the disk surface density.

It is observed in our models that the MF has an impact in the morphology and shape of the swept gas. In the magnetized case, the swept gas shows a smooth structure with denser gas surviving at higher $z$, similar to the results of Tonnesen \& Stone (2014); while in the two non-magnetized models, the gas located above the midplane has a clumpy and filamentary shape. The morphology of the swept gas in the HD and heavy models is due to the equation of state of the gas. In the case where an isothermal equation of state is implemented, like in the setup we presented, the gas is more compressible compared to an adiabatic or magnetized gas (with an adiabatic index $\gamma>1$ ). In our isothermal models, when the wind hits the galaxy, the gas disk is compressed so that clump-like regions form, leading to the development of eddies due to instabilities in the gas and when the eddies are pushed upwards by the wind they generate a tail. This behavior of the gas is similar to the flow of the cigarette smoke, giving the filamentary and clumpy shape to the swept gas in our HD simulation.

It is noticeable that, in some aspects, the swept gas in our MHD model resembles the HI distribution of the spiral galaxy NGC 4522, a galaxy considered a classic example of RPS (see the figure 2 from Kenney et al. 2004): the HI distribution is asymmetric with respect to the stellar disk, is cap shaped, the gas contours are compressed in the upstream side, and it is concave or curved to the downstream side. On the other hand, the stripped gas is not as far from the
NGC 4522 disk as the gas distribution in our MHD model at $t=500 \mathrm{Myr}$.

\subsection{Gunn-Gott criterion}

The Gunn-Gott criterion (GG, Gunn \& Gott 1972) estimates the radius at which a disk galaxy, experiencing the ram pressure face-on, will be truncated. This is determined by equalizing the ram pressure $P_{\text {ram }}=\rho_{\mathrm{ICM}} v_{\mathrm{ICM}}^{2}$ exerted by the wind and the gravitational restoring force in the disk, which is the product of the gravitational force of the galaxy and the surface density of the gas disk $F(r) \Sigma(r)$, that is, the truncation radius is defined by the position where $P_{\mathrm{ram}}=F(r) \Sigma(r)$

\subsubsection{Disk surface density}

In order to verify that our simulations satisfy the GG criterion, we estimate the truncation of our disks measuring the surface density in the $z$ direction. We did these calculations over time also to study the differences in the stripping rate for the three models. Figure 5 shows the evolution of the disk surface density $(\Sigma)$ over time obtained for $|z| \leq 5 \mathrm{kpc}$, for the MHD model, and $|z| \leq 3 \mathrm{kpc}$ for the HD and heavy disks. The differences in the $\Sigma$ integration range in the $z$-direction are due to the different thickness of the MHD with respect to the HD and the heavy ones. We define the truncation radius as the one where $\Sigma$ decays abruptly. At $t=90 \mathrm{Myr}$, the disks are barely perturbed, as can be seen by comparing with figure 4 , and their surface density distribution is similar to the initial condition: the surface density decreases slowly in $r$, and decays rapidly at $r \sim 21-22 \mathrm{kpc}$, except for the MHD disk where the decay is less abrupt. Given that in the MHD case $\Sigma$ decreases approximately two orders of magnitude $\left(10^{-4}-10^{-6} \mathrm{gr} \mathrm{cm}^{-2}\right)$ in $r>20 \mathrm{kpc}$, to obtain the truncation radius of the disk we took the midpoint for this range of densities, in the log-scale, and then we found the value of $r$ where we have this density $\left(\Sigma=10^{-5} \mathrm{gr} \mathrm{cm}^{-2}\right)$, giving $r \sim 23 \mathrm{kpc}$. As mentioned before (see figure 2), the MHD disk has a higher surface density than the HD disk by approximately one order of magnitude due to the extra support that the MF provides. By construction, the heavy disk has a value of $\Sigma_{\text {heavy }} \sim 1.5 \Sigma_{M H D}($ see $\S 2.1)$, but the decay of $\Sigma_{\text {heavy }}$ is more abrupt than the MHD case and lies between the range of $r=21-22 \mathrm{kpc}$, similarly to the HD model. At $t=190 \mathrm{Myr}$, the surface density in the inner MHD disk is still similar to the previous snapshot, but $\Sigma$ decreases more rapidly with radius than at $90 \mathrm{Myr}$, resulting in a disk with $r \sim 19 \mathrm{kpc}$, which is a clear sign that the ICM has started to erode the gas of the disk. At this same time, for the HD run, the disk has been eroded more efficiently than the magnetized one, with $\Sigma_{H D}$ showing an abrupt drop at $r \sim 6 \mathrm{kpc}$. The heavy disk shows a truncation radius of $r \sim 20 \mathrm{kpc}$ and is also evolving similarly to the MHD run.

When the simulations have reached a time of $310 \mathrm{Myr}$, is clear to see that the surface density profile has changed for the MHD and heavy disks, due to the accelerating wind that swepts the gas of these galaxies. This is observed in the fall of the density and the smaller radial extension of the disks, which have been reduced considerably to $r \sim 15 \mathrm{kpc}$ for the MHD and heavy models. The HD model evolves faster in 

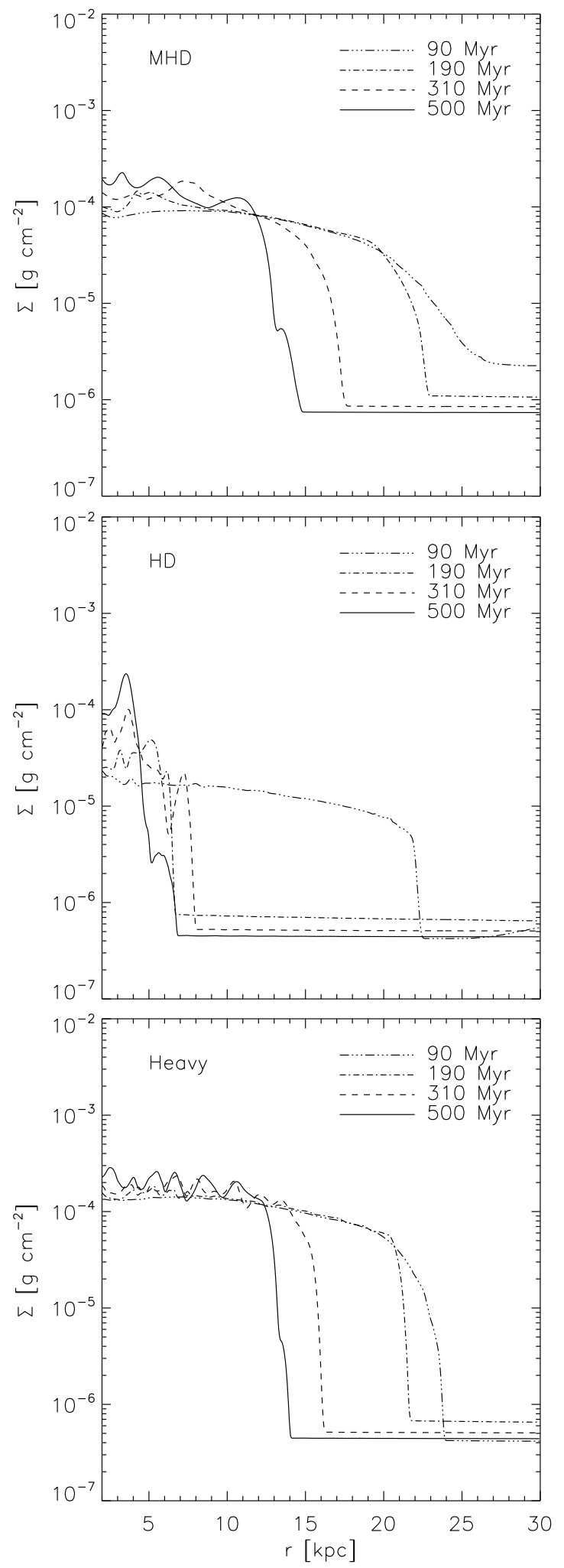

Figure 5. Evolution of surface density for the MHD (top), the HD (middle) and the heavy (bottom) models as a function of galactocentric radius. The surface density is calculated up to $z=$ $\pm 5 \mathrm{kpc}$ from the midplane for the MHD disk and $z= \pm 3 \mathrm{kpc}$ for the HD and heavy disks. The MHD and heavy disks (top and bottom) are eroded more slowly than the HD (middle).

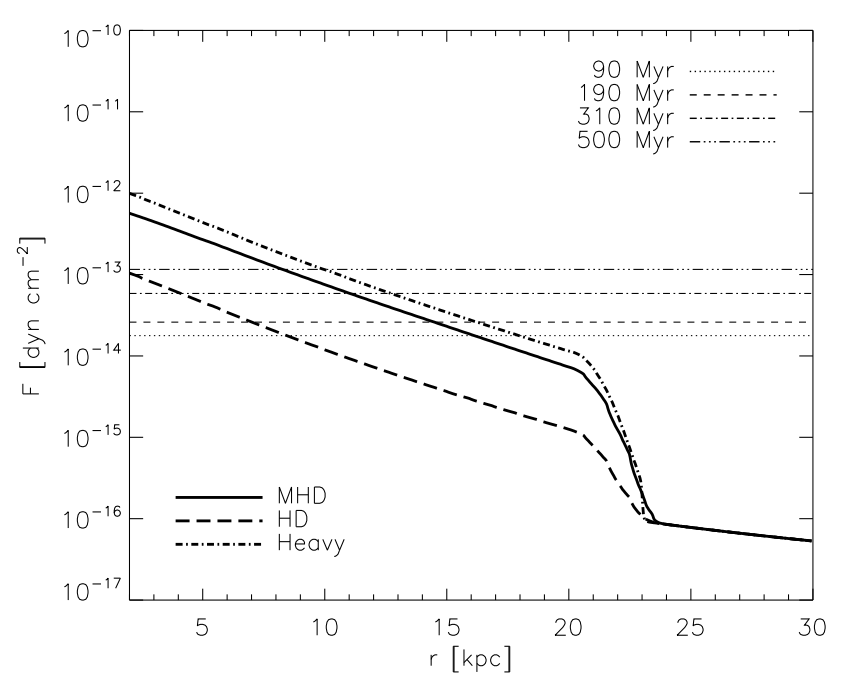

Figure 6. The Gunn-Gott criterion (Gunn \& Gott 1972) for disk gas stripping applied to the simulation disks. The thick lines represent the restoring force of the MHD (solid line), the HD (dashed line) and the heavy (dot-dashed line) disk, while the horizontal lines correspond to the ram pressure measured at different times. For a given set of wind parameters, the radius where the $P_{\text {ram }}$ intersects the restoring force defines the truncation of the gas disk.

time than the other models, as expected, since most of its disk was swept at earlier times $(t=190 \mathrm{Myr})$, presenting a gaseous disk with $r \sim 7 \mathrm{kpc}$.

The ICM wind keeps eroding the gaseous disks of the three models until the end of the simulation $(t=500 \mathrm{Myr})$, leaving a remnant disk with $r \sim 12 \mathrm{kpc}$ and $r=10-12 \mathrm{kpc}$ for the MHD and heavy models, respectively. The HD simulation was run longer, but the disk length reaches an approximate steady truncation radius of $\sim 4 \mathrm{kpc}$ at $t=500 \mathrm{Myr}$, showing that the erosion of this model was faster and more efficient than in the MHD and heavy disks, that loose their gas at a slower rate, as mentioned in $\S 3.1$, and whose disks are truncated at a larger radius.

It is worth mentioning that the increase in $\Sigma$ is related with the following numerical factors. First, is the difficulty of modeling a Cylindrical system in a Cartesian grid. The gas fluxes across grid boundaries in this mismatch lead to errors when the curvature of the circular orbits is large, generating spurious radial flux and a lack of proper rotational support. Second, the rapidly changing gravitational potential in the central regions of the galaxy. The HD disk has a scale height of $\sim 200 \mathrm{pc}$ or even smaller at $r=0$, and with the best spatial resolution achieved just a few grid points are calculating the hydrostatic. We tested how much our models deviate from equilibrium by performing simulations of isolated MHD and HD disk and found that the ill-resolved hydrostatics and rotation generates a collapse of material in the centre of the galaxy, which yields to an increase in the surface density. In the HD isolated disk, the surface density increases from 1 to 2 orders of magnitude in $r<2 \mathrm{kpc}$ from $t=0$ to $t=500 \mathrm{Myr}$. There is also an infall of material in the isolated MHD model, but since this disk is more extended in the z-direction, the increase in the surface density 
is less than one order of magnitude for the same radii and time of evolution compared with the HD case, this is because the grid effects are smaller in the MHD model. When the wind is on, the increase of the surface density is lower than in the isolated cases, since the interaction with the wind diminish this effect. With this in mind, the surface density is not an adequate measure of the inflow of gas derived from the oblique shocks in our models (see §3.3).

\subsubsection{Disk truncation}

Figure 6 shows the gravitational force per unit area for the MHD (solid thick line), the HD (dashed thick line) and the heavy (dot-dashed thick line) disks, approximated as follows: using the gravitational potential of the background axisymmetric model we obtained the maximum force in the $z$ direction as function of $r$ and multiplied by the surface density $\Sigma(r)$ :

$F(r)=F_{z \max }(r) \Sigma(r)=-\frac{\partial \Phi\left(r, z_{\max }\right)}{\partial z} \Sigma(r)$,

where $z_{\max }$ is the point where the gravitational force is maximal. Notice that the gravitational potential is the same for all models but the differences in the restoring force are due to the initial surface density in the disks (see $\S 2.1$ ).

This gravitational restoring force is compared with the ram pressure $P_{\text {ram }}=\rho_{\mathrm{ICM}} v_{\mathrm{ICM}}^{2}$ exerted by the wind (represented with the horizontal lines in the figure). The gravitational force of the disk decreases with increasing radius so, for a given set of parameters for the wind, we expect that the disks are truncated at the radius where both forces are equal, that is, where the $P_{\text {ram }}$ and the force lines cross each other. For the wind parameters, we have $n=10^{-5} \mathrm{~cm}^{-3}$ and the velocity is taken from the simulation. Since it increases in time we chose the value of $v_{\mathrm{ICM}}$ at $z=0$, when it has reached the disk midplane. The lines for the ram pressure are labeled according to the time at which the wind velocity was calculated.

Following the GG criterion, the truncation radius expected for the MHD, HD, and heavy disks is $r \sim 16 \mathrm{kpc}$, $\sim 8.5 \mathrm{kpc}$ and $\sim 18 \mathrm{kpc}$, respectively, with the wind velocity measured at $t=90 \mathrm{Myr}$, which is smaller compared to the cut in the radial direction of our disks measured in the simulation $\left(r_{M H D} \sim 23 \mathrm{kpc}\right.$ and $\left.r_{H D \text {, heavy }} \sim 21-22 \mathrm{kpc}\right)$. At $t=190 \mathrm{Myr}$, the GG truncation radii are also smaller for the MHD and heavy disks compared to the ones calculated from the simulation: $14.5 \mathrm{kpc}$ ( $19 \mathrm{kpc}$ in the simulation) for the MHD and $16.5 \mathrm{kpc}(20 \mathrm{kpc}$ in the simulation) for the heavy model. For the HD disk, the truncation radius measured from the simulation is in better agreement with the one predicted by GG, $r \sim 6-7 \mathrm{kpc}$, and could be due to the fact that this model loses its gas faster than those with a higher initial $\Sigma$.

At later times, from $t=310$ to $500 \mathrm{Myr}$, the truncation radius from GG is more similar to the observed in the simulations, showing slight differences of $1-2 \mathrm{kpc}$ in the two non-magnetized models. By the end of the simulation, $t=500 \mathrm{Myr}$, the radius of the MHD disk should be $r \sim 8-9 \mathrm{kpc}$ according to GG, while the size measured is $r \sim 12 \mathrm{kpc}$. For the HD model, GG predicts $r \sim 2 \mathrm{kpc}$ while we measure $r \sim 4 \mathrm{kpc}$ in the simulation. Finally, in
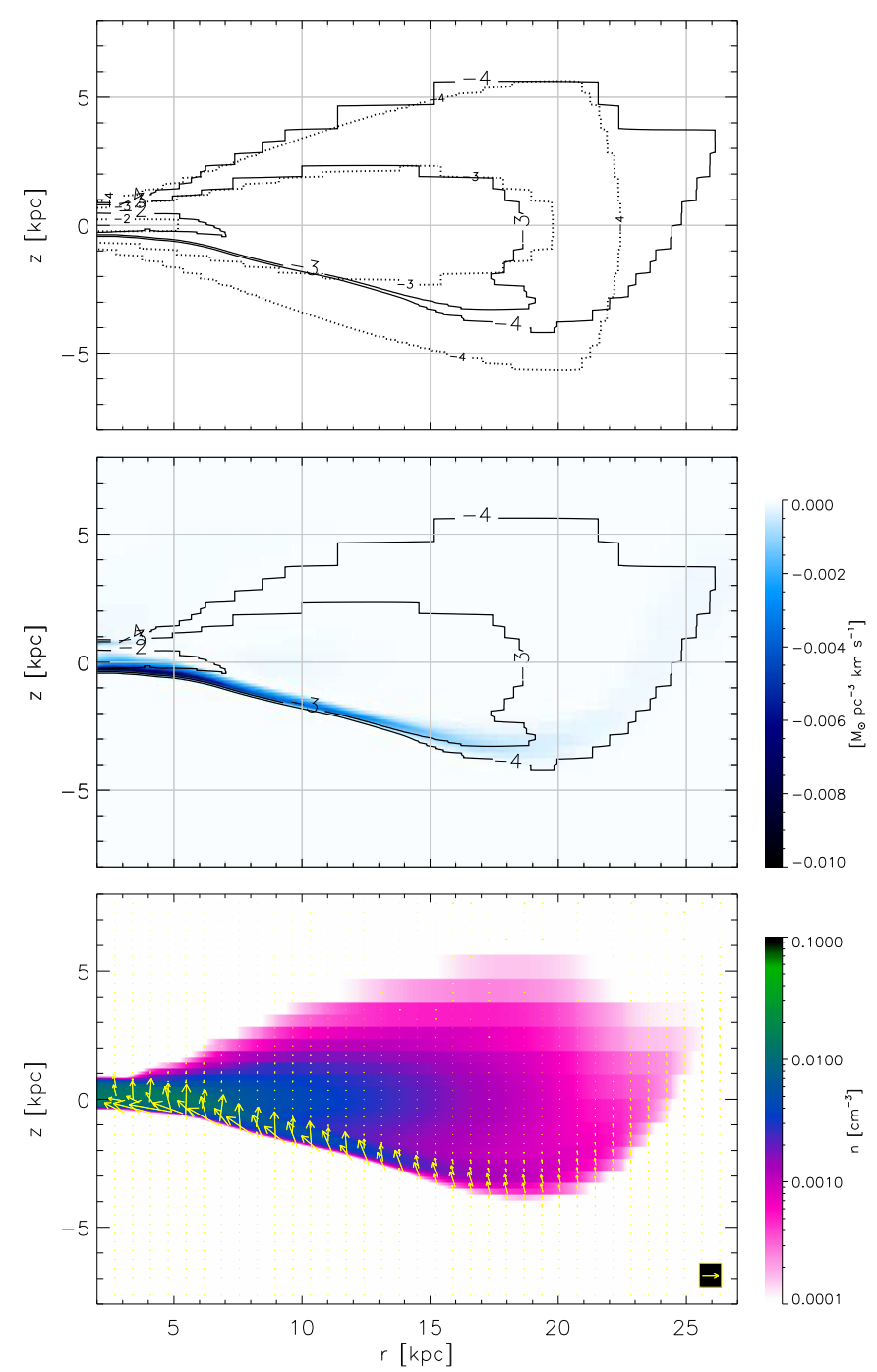

Figure 7. Top: Slice of density for the MHD disk in a $y=0$ cut at time $t=0 \mathrm{Myr}$ (dotted line) and $t=90 \mathrm{Myr}$ (solid line). The contour levels are $n=10^{-4}, 10^{-3}$ and $10^{-2} \mathrm{~cm}^{-3}$ and are labeled from outside-in. Middle: Radially inward flux of mass due to the oblique shocks at the ICM-ISM interaction zone for the same timestep. Colours in the flux map show radially inward mass flux, while the contours are the same as the ones for $t=90 \mathrm{Myr}$ in the top panel, for comparison. Bottom: Slice of density for $t=90$ Myr in a $y=0$ cut, where the colours show the gas number density and flux arrows are overlaid. The scale in the bottom right box corresponds to a mass flux of $3 \times 10^{-3} \mathrm{M}_{\odot} \mathrm{pc}^{-3} \mathrm{~km} \mathrm{~s}^{-1}$. The oblique shocks are produced (due to the "bow tie" shape of the disk) at the disk-wind interface and move the gas from the outer galaxy towards the galactic centre.

the heavy disk we have $r \sim 10 \mathrm{kpc}$ and $r \sim 10-12 \mathrm{kpc}$ with GG criterion and measured in the simulation, respectively. The three models are a reasonable fit to the GG criterion, although the HD and heavy ones are marginally better. This could be due to the assumptions of GG: a zero-width disk (the HD model disk has a scale height of $\sim 200 \mathrm{pc}$ ) and no 


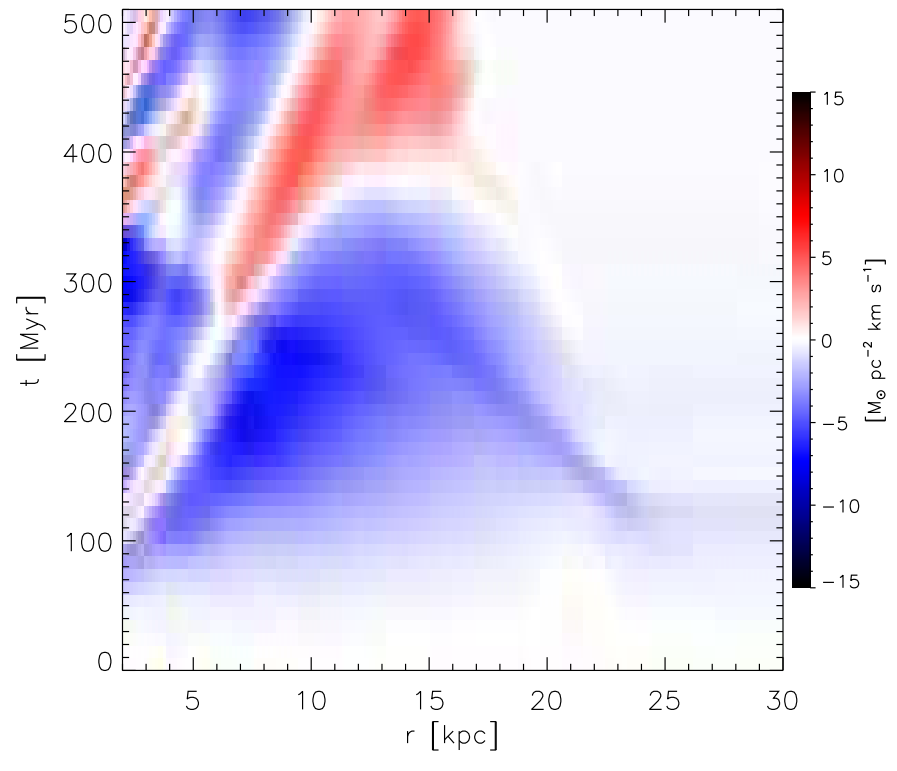

Figure 8. Flux of disk mass integrated in $z$ as a function of time. The radial flow azimuthally averaged is $z$-integrated within the range $|z| \leq 10 \mathrm{kpc}$. The colour-bar shows the inward motions in blue and the outwards as red of the mass flux. The oblique shocks appear in $r=5-10 \mathrm{kpc}$ at $t \gtrsim 100 \mathrm{Myr}$ and increase outwards, driving gas to smaller radii. The shocks reach their maximum strength at $t \approx 250 \mathrm{Myr}$ and after that they start to vanish from the outskirts of the disk $(r>10 \mathrm{kpc})$ when the ram pressure increases and generates an outward flow of gas instead at $t>300 \mathrm{Myr}$.

consideration of the effect of the MF in the gas dynamics. Still, even if the values for the truncation radius do not coincide exactly with the calculations from the simulations, the GG criterion yields a good approximation of how much a gaseous disk may be stripped due to ram pressure.

\subsection{Oblique shocks}

The MHD model has a flared disk (see $\S 2.1$ ) since the MF yields a less-compressive gas layer. Therefore, when the ICM wind reaches the galaxy, an oblique shock is generated in the wind-disk interface. The shocks change the initial distribution of gas in the disk, as can be seen in the density contours in figure 7 (upper panel), and are present in most of the wind-facing side of the disk. The figure compares the initial distribution of the gas density of the disk (dotted line) and at $t=90 \mathrm{Myr}$ (solid line). The most diffuse gas with $n=10^{-4} \mathrm{~cm}^{-3}$ (see the -4 contour) is pushed up and compressed so that the density is more extended in the $+z$ direction than in the $-z$ direction. Conversely, gas with $10^{-3} \mathrm{~cm}^{-3}$ ( -3 contour) is more extended in the $-z$ direction compared to the initial distribution because of the accumulation of the material due to the compression. This compression advances to smaller radii, so that there is more dense gas in the central regions of the galaxy, leading to an expansion of the inner part of the disk with $n=10^{-2} \mathrm{~cm}^{-3}$ $(-2$ contour $)$ in the radial direction and below the midplane in $5 \mathrm{kpc}<r<7 \mathrm{kpc}$.

These oblique shocks lead to a radial inflow of gas toward the inner regions of the galaxy. The middle panel of figure 7 shows this (azimuthally averaged) mass flux at $t=90 \mathrm{Myr}$, with the gas density contours at $t=90 \mathrm{Myr}$ from the upper panel are overlaid. Notice that the inward flux matches with the shocked gas, that is the shocks funnel the gas from larger to smaller radii.

The oblique shocks and the inward flux of mass they produce are present at all radii, being stronger in the inner region of the disk $(2 \mathrm{kpc}<r<10 \mathrm{kpc})$ than in the external region. The inflow of gas can be also observed through flux arrows, which are represented in the bottom panel of figure 7 , where these are overlaid on a density slice as in figure 2 , in the $y=0$ plane also for $t=90 \mathrm{Myr}$. The flux arrows show the motion of gas to the centre of the galaxy produced by the oblique shocks, as we mentioned earlier. The shocked gas at the interface of the disk and the wind $(z<0)$ is pushed up and redirected to smaller radii. These shocks and the inflow of gas from the outskirts $(r>10 \mathrm{kpc})$ may supply the central regions of the disk to ignite star formation or nuclear activity, until the ram pressure increases and starts to sweep the gas from the galaxy.

Figure 8 shows the evolution in time of the $z$-integrated radial gas flux. The gas flux is integrated over a height of $|z| \leq 10 \mathrm{kpc}$. Blue colour represents the radial inflow and red colour is the outflow. As it was previously mentioned, the gas is compressed and funneled to the inner regions of the galaxy. Both the shocks and the flow appear at $t=$ $90-100 \mathrm{Myr}$ and have a radial extension of $r=6-8 \mathrm{kpc}$, where the flux is maximum at this time. As the wind pushes a larger portion of the disk, the shocks and the inflow they produce increase in radius as time increases. For example, in $15 \mathrm{kpc}<r<20 \mathrm{kpc}$ the inflow is active from $t \sim 150 \mathrm{Myr}$ to $t \sim 300-350 \mathrm{Myr}$, which means that the oblique shocks can funnel the gas from the outskirts of the disk to smaller radii before the wind sweeps it out.

The strongest inflow is generated in the time interval of $\sim 100-250$ Myr after the shocks appear, that is, in the $t \sim 200-350$ Myr mark in figure 8. After $t=300 \mathrm{Myr}$, the inflow from the outskirts, that is, the gas originally located in $r>10 \mathrm{kpc}$, becomes weaker until it starts to vanish at $t \sim 400$ Myr. This happens when the wind accelerates and surpasses the galactic gravitational potential, generating an outward flow instead and finally removing the gas of the disk. The swept gas is represented by the red area near the end of the simulation, $t>400 \mathrm{Myr}$ and in the radial range of $r=10 \mathrm{kpc}$ to $r=17 \mathrm{kpc}$. Even though at times $t>\sim$ $350 \mathrm{Myr}$ for radii $r<10 \mathrm{kpc}$, there is still an inflow of gas to the centre of the disk, the motion in this region is more random or disordered due to the interaction with the highspeed wind $\left(v_{\mathrm{ICM}}>700 \mathrm{~km} \mathrm{~s}^{-1}\right)$, which is observed in the blue and red bands. Additionally, the initial flaring of the disk has almost vanished since the wind has compressed and displaced the gas below the midplane.

It can be seen from figure 2 that the heavy disk is also flared but to a lesser extent than the MHD one. We also analyze the oblique shocks in the heavy disk model. We observed an increase in the density of the disk due to a compression of the gas in the wind-disk interaction zone, as in the MHD model. Nevertheless, the layer of the shocked gas is less prominent, with a thickness of $\sim 200 \mathrm{pc}$ which is $\leq 0.4$ of the shocked region in the MHD case, where the thickness of the compressed gas ranges from $\sim 500 \mathrm{pc}-1 \mathrm{kpc}$ in some regions of the disk. The intensity of the azimuthally aver- 
aged flux (as a function of $r$ and $z$ ) for the heavy disk has nearly the same maximum value reached in the MHD case at the time shown in figure $7(t=90 \mathrm{Myr})$, but the strongest flux in the heavy model is located near the centre of the disk, where our setup is not very reliable due to the grid. Studying the flux arrows for the heavy disk we also observed that the vertical motion of the gas in the $+z$ direction dominates over the radial one, that is the wind mostly moves the gas upwards before funneling it to the centre of the galaxy. Addiotionally, the compressed layer of gas is closer to the galactic midplane, so the shock is less oblique. The inflow of gas as a function time for the heavy model is on average a factor of 0.5 lower than in the MHD since in the latter there is gas located at higher $\mathrm{z}$ and therefore, when the flux is integrated in the $\mathrm{z}$ direction, there is more gas moving towards the centre of the disk and the total flux as a function of time and $\mathrm{r}$ is higher. The strongest inflow in the heavy model is present between $t \sim 150-300 \mathrm{Myr}$, lasting $\sim 150 \mathrm{Myr}$. At $t>300 \mathrm{Myr}$ the motion of gas in the heavy model is more disordered in the inner disk $(r<10 \mathrm{kpc})$ until it is finally removed by the wind.

The inflowing gas driven by the oblique shocks raise the possibility of a strong star formation episode in the central part of the galactic disk while the outskirts of the galaxy are being stripped of gas. Observations suggest that S0 galaxies had their last star formation burst in their bulge (Prochaska Chamberlain et al. 2011; Sil'chenko 2006; Sil'chenko et al. 2012; Bedregal 2012; Johnston et al. 2012, 2014, but see Katkov et al. 2015), so this mechanism can provide the central regions with the gas necessary for that burst. Additionally, galaxies undergoing RPS have shown unusual nuclear activity, possibly because the gas is being pushed to the centre and also an enhanced star formation in the region where the gas is compressed by the ICM, that is, the star formation is induced and enhanced by the ram pressure (Cayatte et al. 1990; Poggianti et al. 2016). Poggianti et al. (2017), found a very high incidence of AGN (Seyfert 2) among jellyfish galaxies from MUSE data and they conclude that ram pressure triggers the AGN activity.

There are several points that need to be kept in mind when comparing our simulations with the above quoted results. First, in this work we present only a generic model for a flared disk galaxy. More studies must be performed in order to verify the presence of these oblique shocks in galaxies. In our model, the flare is created by a magnetic field, but this is not the only mechanism to create such a disk (for example, a different equation of state for the gas as presented by Roediger \& Hensler 2005). The second point to consider is that the central regions of the disk in our simulation are too idealized, and so it is hard to state how much of the inward flux created by the shock actually reaches the centre of the galaxy. Also, the perfectly face-on geometry of the interaction might have an influence of the shocked-gas galactic inflow. More numerical experiments, with less idealized conditions, will be presented in future contributions. Nevertheless, as long as the disk flares, oblique shocks should appear for a face-on ICM wind interaction and the presence of a magnetic field is a good mechanism to generate such a flare. Also, since a magnetized disk is less compressible than a pure HD one, the shocked layer in the MHD model will be more pressurized and will try to drain gas, either to the outskirts and/or to the central regions of the disk.

\section{CONCLUSIONS}

We performed MHD and HD simulations of a disk galaxy subject to RPS to analyze the impact of the MF in the dynamics of the gas during the stripping event. Both models were set up in hydrostatic equilibrium with the gravitational potential of an M33-like galaxy, without the galactic bulge component of the potential.

We found that the galactic MF gives us a thicker gaseous disk than the HD one, which change the dynamics of the model, that is, we have gas farther from the galactic potential well (in the $z$ direction) in the MHD, plus the surface density in $z$ is higher than in an HD disk with the same midplane density. When the ICM wind hits the disks, at the beginning of the simulation, the MHD disk is hardly affected by the wind, since no significant changes were observed in the initial shape of the disk, only the compressed gas in the interaction interface. The HD disk is easily perturbed and pushed off the galactic midplane by the wind. Then the gravitational potential pulls back the material to disk, generating an infall of gas to the disk until the ram pressure exceeds the gravitational force and removes the gaseous disk.

The evolution of both models continues as the wind velocity increases. Their ISM is removed of the disk, from the outside-in, and reaches higher $z$ above the midplane. When the models have evolved for $t=500 \mathrm{Myr}$, the swept gas in the MHD case is denser, reaches a height of approximately $z \sim 20 \mathrm{kpc}$, and the disk has been truncated to $r \sim 10 \mathrm{kpc}$. In the HD run, the swept gas is farther away from the galactic midplane, $z \sim 40 \mathrm{kpc}$, and has a lower density than the MHD. The disk is also eroded to a smaller radius of $r \sim$ $4-5 \mathrm{kpc}$. These results show that the removal of the gas disk is less efficient in the MHD model than in the HD case with the same midplane density.

The main differences found so far between the models are:

- The HD disk is more easily eroded than the MHD one, because in the magnetized case we have a higher surface density $\Sigma$ and the gas is less compressible than in the HD model. Since the surface density strongly affects the stripping rate, we developed an HD model with approximately the same $\Sigma$ as the MHD, which shows a similar stripping rate. This "heavy" HD disk has a very high midplane volumetric density that makes it unrealistic.

- The swept gas for the MHD model has a smooth appearance whilst for the HD models (both the regular and the heavy disks), the gas above the galaxy has a clumpier and filamentary-like morphology, that is, the MF mainly affects the shape and structure of the swept gas.

Previous RPS simulations have obtained broader tails, that is the swept gas of the disks, compared with observations of jellyfish galaxies (galaxies undergoing RPS). It was expected that additional physical properties, such as MF, cooling, star formation, etc. may help to solve this problem, presenting narrower tails in the simulations. Ruszkowski et al. (2014) presented MHD simulations with radiative cooling and self-gravity for a magnetized ICM only, 
and showed that the MF can give narrower gas tails compared with HD models. Our runs show the opposite behavior, the swept gas from the disk in the MHD model is broader than the HD, but we do not have the same initial set-up as them. The differences in the tail width could be also accounted for the radiative cooling. HD simulations performed by Tonnesen \& Bryan (2010) including radiative cooling showed narrower tails in better agreement with observations, compared to non-cooling models. On the other hand, the swept gas from our MHD model shows a smooth structure, while the HD models looks clumpier, similarly to tails observed in Ruszkowski et al. (2014). The differences observed in the shape and morphology of the swept gas in our models lie in the equation of state of the gas, that is an isothermal gas is more compressible than an adiabatic (e.g. Roediger \& Hensler 2005; Roediger \& Brüggen 2007, 2008; Tonnesen \& Bryan 2009, 2010) or a magnetized gas, and when the wind hits the galaxy clump-like regions appear in our HD simulations. When these regions are pushed and eroded by the wind, they generate tails in the swept gas, where the flow is similar to the cigarette smoke, giving the filamentary and clumpy shape to the swept gas in our HD simulation. A more detailed analysis of the morphology and structure of the gas tails will be presented in the near future (Ramos-Martínez et al. in preparation). Tonnesen \& Stone (2014) also performed RPS models with galactic MF, with different configurations and intensities for the field, and they found that the MFs do not make a significant difference in the stripping rate of ISM, but the MF inhibits the mixing of the gas tail with the surrounding ICM and unmixed gas survives at larger distances from the disk. In our results we see a similar trend, since the swept gas in the MHD model also remains unmixed for longer time, despite the fact that the $z$-height is smaller compared to our HD run. The differences in the tail appearance and structure for their MHD and HD models is not so evident or dramatic. Since the approach of our models is not the same as Ruszkowski et al. (2014) and Tonnesen \& Stone (2014) we cannot make an analytical comparison with their works. We consider that, in order to understand if MF can make a significant difference and its relevance in the interaction of the ICM-ISM, further investigation will be needed.

Even when our HD simulation ran for $1 \mathrm{Gyr}$, the model reached equilibrium at $t \lesssim 500 \mathrm{Myr}$ : the truncated disk remained with the same radius although the wind was still accelerating to a maximum velocity of $1000 \mathrm{~km} \mathrm{~s}^{-1}$ before the simulation ended. Therefore, we can assume that the MHD run has also reached equilibrium with the ram pressure, or is near to it. The remaining gaseous disk could be removed by other mechanism, like interactions or fly-by's with other galaxies (e.g. galaxy harassment), this should be taken into account because these objects are not completely isolated, specially in clusters. Interactions between galaxies can remove the gas or trigger star formation so the ISM is consumed or exhausted.

It is well known that RPS works well removing the gas of the galaxies, but this process fails in reproduce other S0s features, like higher bulge-to-disc ratios than spirals, given that RPS has been proposed as a transformation mechanism of spirals to S0s. For our magnetized case, with inefficient RPS, we found an interesting behavior in the gas: there are motions of gas from large radii to the galactic cen- tre. This phenomenon occurs only in the early stages of the simulation, when the wind hits the disk, and it is produced by oblique shocks at the interface of the interaction. The oblique shocks appear because of our flared gas disk due to the MF presence and lead the gas to the centre of the disk, which may help to maintain a reservoir of gas available for star formation in the central region of the galaxy, which in consequence could produce a thicker bulge that may lead to a higher bulge-to-disk ratio. Studies have shown that the last star formation burst in S0s galaxies took place in the bulge (Prochaska Chamberlain et al. 2011; Sil'chenko 2006; Sil'chenko et al. 2012; Bedregal 2012; Johnston et al. 2012, 2014, but see Katkov et al. 2015). Besides, if new stars are born from the remaining gas in the centre, their strong winds could expel the rest of the ISM from the galaxy.

Other observations of galaxies affected by RPS have shown unusual nuclear activity, that is, the gas may be pushed to the centre and the compression produced by the ICM enhances star formation: the star formation is induced and enhanced by the ram pressure (Cayatte et al. 1990). Poggianti et al. (2016) showed an atlas of stripping candidates where most of their galaxies presented higher star formation compared to non-stripped galaxies. From this results, the oblique shocks can be seen as a mechanism that enhance the formation of new stars in the remaining disk or even trigger nuclear activity (e.g. an AGN). Also Poggianti et al. (2017), found a very high incidence of AGN (Seyfert 2) among jellyfish galaxies from MUSE data and the conclusion is that ram pressure triggers the AGN activity. Since the flux of gas derived from the oblique shocks in our MHD simulation lasted about $\sim 150$ Myr from the time the wind hit the disk, this could be considered as comparable with the duty cycle of AGN's, which has been estimated in 10-100 Myr (Haehnelt \& Rees 1993), but given that our simulation does not properly model the central regions of the galaxy, nor we have a central black hole, we can only speculate that the oblique shocks will transport the gas for enough time to ignite an AGN. Other tests need to be performed to better study the funneling of gas towards the central regions of the galaxy, such as different wind profiles and angles, and different disk surface densities and flare strengths.

Additionally, it has been reported that the star formation can continue in the tail of the stripped gas, as it is shown in observations of HII regions in the tail of a galaxy subject to RPS (Kenney \& Koopmann 1999; Boselli \& Gavazzi 2006; Cortese et al. 2007; Yoshida et al. 2008; Hester et al. 2010; Sun et al. 2010; Yagi et al. 2010; Abramson et al. 2011; Kenney et al. 2014; Poggianti et al. 2016). Due to the limitations of our models, not enough resolution nor the appropriate equation of state to solve the star formation, we cannot explore the possibility of new stars born in the swept gas of our models or the centre of the disks from the motions of gas originated from the oblique shocks. More on this subject will be done in a future work as well as an in-depth analysis of the swept gas for the MHD model (Ramos-Martínez et al. in preparation).

\section{ACKNOWLEDGMENTS}

We thank B. Poggianti, M. Owers, and P. Appleton for useful comments and discussions. We also thank an anonymous ref- 
eree for comments that helped improve this manuscript. We acknowledge financial support from UNAM-DGAPA PAPIIT grant IN100916, and CONACyT for support for MRM.

\section{REFERENCES}

Abadi, M. G., Moore, B. \& Bower, R. G. 1999, MNRAS, 308, 947 Abramson, A. \& Kenney, J. D. P. 2014, AJ, 147, 63

Abramson, A., Kenney, J. D. P., Crowl, H. H., Chung, A., van Gorkom, J. H., Vollmer, B. \& Schiminovich, D. 2011, AJ, 141, 164

Abramson, A., Kenney, J., Crowl, H., \& Tal, T. 2016, AJ, 152, 32

Adebahr, B., Krause, M., Klein, U., Weżgowiec, M., Bomans, D. J. \& Dettmar, R. J. 2013, A\&A, 555A, 23A

Aguerri, J., Balcells, M. \& Peletier, R. 2001, A\&A, 428

Aguerri, J., Agulli, I., Diaferio, A. \& Dalla Vecchia, C. 2017, MNRAS, 468,364

Allen, C. \& Santillán, A. 1991, Rev. Mex. Astron. Astrofis., 22, 255

Beck, R. in The magnetized plasma in galaxy evolution 2005, ed. K. T. Chyży, K. Otmianowska-Mazur, M. Soida \& R.-J. Dettmar, 193

Beck, R., Fletcher, A., Shukurov, A., Snodin, A., Sokoloff, D. D., Ehle, M., Moss, D. \& Shoutenkov, V. 2005, A\&A, 444, 739B

Beck, R. \& Wielebinski, R. in Planets, Stars and Stellar Systems 2013, Vol. 5, ed. G. Gilmore, Springer

Bedregal, A. G. 2012, A\&AT, 27, 177

Bekki, K., Couch, W. J. \& Shioya, Y. 2002, ApJ, 577, 651

Bekki, K. \& Couch, W. J. 2003, ApJ, 596, L13

Bekki, K. 2014, MNRAS, 438, 444

Biviano, A. \& Katgert, P. 2004, A\&A, 424, 779

Borlaff, A., Eliche-Moral, M. C., Rodríguez-Pérez, C., Querejeta, M., Tapia, T., Pérez-González, P. G., Zamorano, J., Gallego, J. \& Beckman, J. 2014, A\&A, 570, 103

Boselli, A. \& Gavazzi, G. 2006, PASP, 118, 517

Boselli, A., Cortese, L., Boquien, M., Boissier, S., Catinella, B., Gavazzi, G., Lagos, C. \& Saintonge, A. 2014, A\&A, 564, A67

Butcher, H. \& Oemler, Jr., A. 1978, ApJ, 219, 18

Cayatte, V., van Gorkom, J. H., Balkowski, C. \& Kotanyi, C. 1990, AJ, 100, 604

Chung, E. J., Rhee, M.-H., Kim, H., Yun, M. S., Heyer, M. \& Young, J. S. 2009, ApJS, 184, 199

Chyży, K. T. \& Beck, R. 2004, A\&A, 417, 541C

Corbelli, E. 2003, MNRAS, 342,199

Cortese, L., Marcillac, D., Richard, J., Bravo-Alfaro, H., Kneib, J.-P., Rieke, G., Covone, G., Egami, E., Rigby, J., Czoske, O. \& Davies, J. 2007, MNRAS, 376, 157

Dressler, A. 1980, ApJ, 236, 351

Dressler, A. 1986, ApJ, 301, 35

Dressler, A., Oemler, A., Smail, I., Barger, A., Butcher, H., Poggianti, B. M. \& Sharples, R. M. 1997, ApJ, 490, 577

Elmegreen, D. M., Elmegreen, B. G.,Frogel, J. A., Eskridge, P. B., Pogge, R. W., Gallagher, A. \& Iams, J. 2002, AJ, 124, 777

Farouki, R. \& Shapiro, S. L. 1980, ApJ, 241, 928

Fasano, G., Poggianti, B. M., Couch, W. J., Bettoni, D, Kjærgaard, P. \& Moles, M. 2000, ApJ, 542, 673F

Fletcher, A. 2010, ASPC, 438, 197F

Fletcher, A., Beck, R., Shukurov, A., Berkhuijsen, E. M., Horellou, C. 2011, MNRAS, 412, 2396

Fossati, M., Fumagalli, M., Boselli, A., Gavazzi, G., Sun, M. \& Wilman, D. J. 2016, MNRAS, 455, 2028

Frick, P, Stepanov, R, Beck, R, Sokoloff, D., Shukurov, A., Ehle, M. \& Lundgren, A. 2016, A\&A, 585, A21

Fuchs, B. \& von Linden, S. 1998, MNRAS, 294, 513

Fumagalli, M., Fossati, M., Hau, G. K. T., Gavazzi, G., Bower, R., Sun, M. \& Boselli, A. 2014, MNRAS, 445, 4335
Gallagher, J. S., Faber, S. M. \& Balick, B. 1975, ApJ, 202, 7G

Gießübel, R. 2012, PhD Thesis, University of Cologne

Giraud, E. 1986, A\&A, 167, 25

Gómez, G. C. \& Cox, D. 2002, ApJ, 580, 235

Gunn, J. E. \& Gott, J. R. I. 1972, ApJ, 176, 1

Haehnelt, M. G. \& Rees, M. J. 1993, MNRAS, 263, 168H

Hester, J. A., Seibert, M., Neill, J. D., Wyder, T K., Gil de Paz, A., Madore, B. F., Martin, D. C., Schiminovich, D. \& Rich, R. M. 2010,ApJ, 716L, 14

Icke, V. 1985, A\&A, 144, 115

Jáchym, P., Combes, F., Cortese, L., Sun, M. \& Kenney, J. D. P. 2014, ApJ, 792, 11

Jáchym, P., Köpen, J., Palouš, J. \& Combes, F. 2009, A\&A, 500, 693

Johnston E. J., Aragon-Salamanca A., Merrifield M. R. \& Bedregal A. G. 2012, MNRAS, 422, 2590

Johnston E. J., Aragon-Salamanca A. \& Merrifield M. R. 2014, MNRAS, 441, 333

Kapferer, W., Kronberger, T., Ferrari, C., Riser, T. \& Schindler, S. 2008, MNRAS, 389, 1405

Katkov, I. Y., Kniazev, A. Y. \& Sil'chenko, O. K. 2015, AJ, 150, 24

Kenny, J. D. P. \& Koopmann, R. A. 1999, AJ, 117, 181

Kenney, J. D. P., van Gorkom, J. \& Vollmer, B. 2004, AJ, 127, 3361

Kenney, J. D. P., Geha, M., Jáchym, P., Crowl, H. H., Dague, W., Chung, A., van Gorkom, J. \& Vollmer, B. 2014, ApJ, 780, 119

Klein, U., Weiland, H. \& Brinks, E. 1991, A\&A, 246, 323K

Koopmann, R. A., \& Kenney, J. D. P. 2004, ApJ, 613, 866

Kronberger, T., Kapferer, W., Ferrari, C., Unterguggenberger, S. \& Schindler, S. 2008, A\&A, 481, 337

Larson, R. B., Tinsley, B. M. \& Caldwell, C. N. 1980, ApJ, 237, 692

Moore, B., Katz, N., Lake, G., Dressler, A. \& Oemler, A. 1996, Nat, 379,613

Niklas, S. 1995, PhD Thesis, University of Bonn

Oemler, A. 1974, ApJ, 194, 10

Otmianowska-Mazur, K. \& Vollmer, B. 2003, A\&A, 402, 879

Pfrommer, C. \& Dursi, J. 2010, Nature Physics, 6, 520

Poggianti, B. M., Fasano, G., Omizzolo, A., Gullieuszik, M., Bettoni, D., Moretti, A., Paccagnella, A., Jaffé Y. L., Vulcani, B., Fritz, J, Couch W. \& D'Onofrio, M. 2016, AJ, 151, 78P

Poggianti, B. M., Jaffé, Y. L., Moretti, A., Gullieuszik, M., Radovich, M., and Tonnesen, S., Fritz, J., Bettoni, D., Vulcani, B., Fasano, G., Bellhouse, C., Hau, G. \& Omizzolo, A. 2017, Nat, 548, 304P

Postman, M. \& Geller, M. J. 1984, ApJ, 281, 95P

Prochaska Chamberlain, L. C., Courteau, S., McDonald, M \& Rose, J. A. 2011, MNRAS, 412, 423P

Quilis, V., Moore, B. \& Bower, R. 2000, Science, 288, 1617

Rengarajan, T. N., Karnik, A. D. \& Iyengar, K. V. K. 1997, MNRAS, 290, 1

Roediger, E. \& Brüggen, M. 2006, MNRAS, 369, 567

Roediger, E. \& Brüggen, M. 2007, MNRAS, 380, 1399

Roediger, E. \& Brüggen, M. 2008, MNRAS, 388, 465

Roediger, E., Brüggen, M. \& Hoeft, M. 2006, MNRAS, 371, 609

Roediger, E. \& Hensler, G. 2005, A\&A, 433, 875

Ruszkowski, M., Brüggen, M., Lee, D. \& Shin, M. S. 2014, ApJ, 784,75

Schulz, S. \& Struck, C. 2001, MNRAS, 328,185

Scodeggio, M. \& Gavazzi, G. 1993, ApJ, 409, 110

Sellwood, J. A. \& Carlberg, R. G. 1984, ApJ, 282, 61

Sil'Chenko, O. 2006, ApJ, 641, 229

Sil'chenko, O., Proshina, I. S., Shulga, A. P. \& Koposov, S. E. 2012, MNRAS, 427, 790

Simien, F. \& de Vaucouleurs, G. 1986, ApJ, 302, 564S

Sivanandam, S., Rieke, M. J. \& Rieke, G. H. 2010, ApJ, 717, 147 
Soida, M., Otmianowska-Mazur, K., Chyży, K. \& Vollmer, B. 2006, A\&A, 458, 727

Stein, Y., Bomans, D. J., Ferguson, A. M. N. \& Dettmar, R.-J. 2017, A\&A, 605, A5

Steinhauser, D., Haider, M., Kapferer, W. \& Schindler, S. 2012, A\&A, 544, A54

Sun, M., Donahue, M., Roediger, E., Nulsen, P. E. J., Voit, G. M., Sarazin, C., Forman, W. \& Jones, C. 2010, ApJ, 708, 946

Sun, M., Donahue, M. \& Voit, G. M. 2007, ApJ, 671, 190

Sun, M., Jones, C. \& Forman, W. 2006, ApJ, 637, L81

Tabatabaei, F. S., Krause, M., Fletcher, A. \& Beck, R. 2008, A\&A, 490, 1005

Tapia, T. et al. 2014, A\&A, 565, 31

Teyssier, R. 2002, A\&A, 385, 337

Tonnesen, S. \& Bryan, G. L. 2009, ApJ, 694, 789

Tonnesen, S. \& Bryan, G. L. 2010, ApJ, 709, 1203

Tonnesen, S. \& Bryan, G. L. 2012, MNRAS, 422, 1609

Tonnesen, S. \& Stone, J. 2014, ApJ, 795, 148

Toomre, A. \& Toomre, J. 1972, ApJ, 178, 623

Vollmer, B., Cayatte, V., Balkowski, C. \& Duschl, W. J. 2001, ApJ, 561, 708

Vollmer, B., Beck, R., Kenney, J. D. P. \& van Gorkom, J. H. 2004, AJ, 127, 3375

Vollmer, B., Braine, J., Pappalardo, C. \& Hily-Blant, P. 2008, A\&A, 491, 455

Vollmer, B., Marcelin, M., Amram, P., Balkowski, C., Cayatte, V. \& Garrido, O. 2000, A\&A, 364, 532

Vollmer, B., Soida, M., Beck, R., et al. 2007, A\&A, 464, L37

Vollmer, B., Soida, M., Otmianowska-Mazur, K., Kenney, J. D., van Gorkom, J. H. \& Beck, R. 2006, A\&A, 453, 883

Yagi, M., Yoshida, M., Komiyama, Y., Kashikawa, N., Furusawa, H., Okamura, S., Graham, A. W., Miller, N. A., Carter, D., Mobasher, B. \& Jogee, S. 2010, AJ, 140, 1814

Yoshida, M., Yagi, M., Komiyama, Y., Furusawa, H., Kashikawa, N., Koyama, Y., Yamanoi, H., Hattori, T. \& Okamura, S. 2008, ApJ, 688, 918-930

Zhang, B., Sun, M., Ji, L., Sarazin, C., Lin, X. B., Nulsen, P. E. J., Roediger, E., Donahue, M., Forman, W., Jones, C., Voit, G. M. \& Kong, X. 2013, ApJ, 777, 122

This paper has been typeset from a $\mathrm{T}_{\mathrm{E}} \mathrm{X} / \mathrm{LAT}_{\mathrm{E}} \mathrm{X}$ file prepared by the author. 